\title{
The effects of eccentric training on electromyographic activity and performance in soccer players
}

\author{
Stergios Komsis ${ }^{1}$, Georgios Komsis ${ }^{1}$, Ioannis Gissis ${ }^{1}$, Christos Papadopoulos ${ }^{1}$, Dimitrios Patikas ${ }^{1}$, \\ Lida Mademli ${ }^{1}$, Panagiotis Papadopoulos ${ }^{2}$, Vassilis Paschalis ${ }^{3,4}$, Ioannis S. Vrabas ${ }^{1}$ \\ ${ }^{1}$ Department of Physical Education and Sports Science at Serres, Aristotle University of Thessaloniki, Greece \\ ${ }^{2}$ Department of Physical Education and Sports Science, Aristotle University of Thessaloniki, Greece \\ ${ }^{3}$ Department of Physical Education and Sports Science, University of Thessaly, Trikala, Greece \\ ${ }^{4}$ Laboratory of Exercise, Health and Human Performance, Research Center, European University of Cyprus, Nicosia, Cyprus
}

\section{Email address:}

igkisis@phed-sr.auth.gr (I. Gissis)

\section{To cite this article:}

Stergios Komsis, Georgios Komsis, Ioannis Gissis, Christos Papadopoulos, Dimitrios Patikas, Lida Mademli, Panagiotis Papadopoulos, Vassilis Paschalis, Ioannis S. Vrabas. The Effects of Eccentric Training on Electromyographic Activity and Performance in Soccer Players. American Journal of Sports Science. Vol. 2, No. 2, 2014, pp. 23-29. doi: 10.11648/j.ajss.20140202.13

\begin{abstract}
The purpose of the present study was to investigate the effects of eccentric training using a multi-joint dynamometer, on the electromyographic activity of rectus femoris, biceps femoris and medial gastrocnemius during counter movement jumps, drop jumps as well as during maximal eccentric actions. Sixteen amateur soccer players was divided into equal sized groups, the control group who participated in their regular training and the training group who performed 16 sessions (in 8 weeks) of eccentric exercise using a multi joint isokinetic dynamometer. The performance of counter movement and drop jumps were evaluated on a force plate. Additionally, maximal isometric, concentric and eccentric force were assessed on the isokinetic dynamometer. After the eccentric training, the electromyographic activity during the concentric phase of counter movement jumps was found to be increased in rectus femoris $(p<0.05)$ and reduced in biceps femoris $(p<0.05)$. During drop jumps, electromyographic activity of the experimental group found to be increased in the pre-activation phase of gastrocnemius $(\mathrm{p}<0.05)$. Additionally, during the takeoff phase of the drop jumps smaller angles for hip and ankle joints were observed $(\mathrm{p}<0.05)$. Finally, the electromyographic activity during eccentric strength evaluation were found to be elevated in rectus femoris $(p<0.05)$ and decreased in gastrocnemius $(p<0.05)$. The eccentric training which can cause neural adaptations, faster recruitment of motor units as well as changes in the architecture in muscle tendon system may also cause the alterations in electromyographic activity of leg muscles as observed in the present investigation.
\end{abstract}

Keywords: Contact Time, Isokinetic Dynamometry, Myoelectrical Properties, Soccer players, Stretch-Shortening Cycle, Vertical Jump

\section{Introduction}

During the most human movement there is an active stretch (eccentric action) of the muscles followed by an immediate shortening (concentric contraction) of the same muscles a condition known as the stretch - shortening cycle (SSC). However, it is also well documented that eccentric muscle actions induce muscle damage which cause delayed onset muscle soreness while deterioration in muscle strength and range of movement is observed [1-4]. Fortunately, it is well established that a repeated bout of eccentric exercise results in markedly reduced symptoms of muscle damage than the initial bout $[5,6]$.
It is also known that exercise including eccentric muscle actions is more effective in increasing muscle strength compared to concentric muscle contractions [7], probably due to the fact that fast twitch motor units are selectively activated during eccentric muscle actions [8]. Moreover, neuromuscular adaptations induced by eccentric training have been suggested to cause greater strength improvements than concentric training [9]. Lately, the use of the isokinetic dynamometer in resistance training insures pure eccentric muscle actions of maximal intensity through the whole range of motion of the joint [10]. The features of isokinetic muscle activity induces mechanical overload to muscles which may in turn influence gene expression via mechanotransduction pathways [11], leading in muscle 
hypertrophy, elevation in muscle strength and protection of the muscle against subsequent injury $[12,13]$.

According to electromyographic (EMG) data, voluntary eccentric muscle actions induce simultaneous activation of the agonist and antagonist muscles [14, 15]. Such a neural mechanism decreases the levels of torque production and stabilizes the joint in response to the high muscle tensions induced by eccentric contractions [16]. It is known that the external torque is the resultant of the torque produced by the agonist muscles minus the torque produced by the antagonist muscles [17]. Interestingly, after eccentric training it was observed a decrease in antagonist EMG activity which means less negative torque output resulting in higher overall torque output [17]. However, no study has examined biomechanical and neural factors after exercise training with eccentric muscle actions of maximal intensity, and their effect on the jumping ability. Therefore, the purpose of the present study was to investigate the effects of eccentric training using a multi-joint dynamometer on EMG activity of major lower limb muscles during counter movement jumps, drop jumps as well as during maximal eccentric muscle action.

\section{Material and Methods}

\subsection{Participants}

Sixteen healthy male amateur soccer players with no history of neurological injuries or diseases participated in the present investigation. They were randomly divided in two equal sized groups, the control group (age 22.1 $\pm 0.3 \mathrm{y}$, height $174 \pm 4 \mathrm{~cm}$, and body mass $86.5 \pm 3.5 \mathrm{~kg}$ ) and the eccentric training group (age $24 \pm 0.5 \mathrm{y}$; height $176 \pm 6 \mathrm{~cm}$; and body mass $84.5 \pm 5.5 \mathrm{~kg}$ ). Except for their usual soccer training, subjects did not participate in other organized exercise training programs. They were also instructed not to take anti-inflammatory drugs and abstain from strenuous exercise for 3 days prior to the beginning of the investigation and during the data collection period. Subjects of groups had no experience in organized eccentric training program. The procedures were in accordance with the Helsinki declaration of 1975 , as revised in 2000. An informed written consent approved by the local university Ethics Committee was obtained by all participants after they were informed of all risks, discomforts and benefits involved in the study.

\subsection{Training Protocol}

Participants of the experimental group performed a training protocol using a custom-built multi-joint isokinetic dynamometer (Ydromichaniki SA). The training protocol is described in Table 1. The training protocol was performed during the preseason period. Subjects of the experimental group were performing the sitting leg press exercise two times per week for eight weeks, while between two exercise sessions a recovery period of at least 48 hours was incorporated. During each exercise session, participants were sitting comfortably on the seat of the isokinetic dynamometer placing their legs on a force-plate while their chest and hips were stabilized with straps (Figure 1). During the eccentric exercise sessions the knee joint range of motion was set between $130^{\circ}$ to $160^{\circ}$ and the linear velocity was set at $0.35 \mathrm{~m} / \mathrm{s}$. The first week of the training period served as familiarization period using $70 \%$ of the maximal eccentric performance [18-20]. The intensity of the follow up sessions was constantly controlled and readjusted by performing regular assessments of maximal eccentric performance (Table 1). Before each exercise session, subjects performed a warm-up consisting of 6-min cycling on an ergometer at $70 \mathrm{rpm}$ and $50 \mathrm{~W}$ followed by 5 min of ordinary stretching exercises of the major muscle groups of the lower limbs.

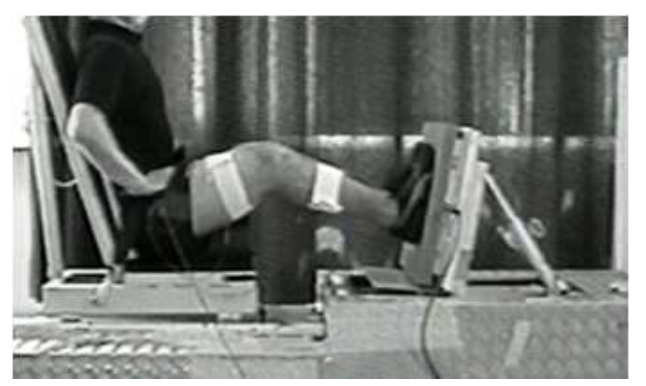

Figure 1. The multi-joint isokinetic dynamometer

\subsection{Performance Assessments}

All the assessments were performed two days before the beginning of the training program and two days after its termination. For the evaluation of strength output the same multi joint isokinetic dynamometer was used. During the assessment 2 trials of isometric maximal strength output (at $120^{\circ}$ knee joint angle) were performed and the subjects were instructed to push as hard as possible the force-plate and maintain the maximum force for about $4 \mathrm{~s}$. For the assessment of concentric and eccentric force output two trials were performed at a constant velocity of $0.35 \mathrm{~m} / \mathrm{s}$ while the range of motion for the knee joint was set at $130-160^{\circ}$. The best performance of each exercise mode was recorded for the statistical analysis.

For the assessment of jumping ability, the subjects performed a series of jumps on a force platform (Kistler 9281CA, Instrumente AG, Winterthur, Switzerland) sampling at $1000 \mathrm{~Hz}$. The participants performed 2 jumps with initial acceleration downwards (counter movement jump) and 2 drop jumps, falling from $30 \mathrm{~cm}$ height while in both conditions their hands were placed on their waist. In counter movement jumps the best vertical performance measured in $\mathrm{cm}$ was recorded while in drop jumps the contact time measured in msec along with the best high performance measured in $\mathrm{cm}$ was recorded. During counter movement jump, subjects were instructed to move downwards as fast as possible and to jump as high as possible. During the drop jump, subjects were asked to contact with the ground fast and to execute a vertical jump as high as possible. A 2 min interval was incorporated 
between the trials. During both counter movement and drop jumps the EMG activity was recorded.

Table 1. Eccentric training protocol. Two exercise sessions were performed each week. Intensity is in $\%$ of $1 R M$.

\begin{tabular}{|c|c|c|c|}
\hline & Sets $\times$ reps & Intensity & Rest (min) \\
\hline $1^{\text {st }}$ week & $3 \times 10$ & 70 & 5 \\
\hline \multicolumn{4}{|c|}{ Evaluation of eccentric power output } \\
\hline $2^{\text {nd }}$ week & $5 \times 8$ & 80 & 3 \\
\hline $3^{\text {rd }}$ week & $5 \times 8$ & 80 & 3 \\
\hline \multicolumn{4}{|c|}{ Evaluation of eccentric power output } \\
\hline $4^{\text {th }}$ week & $4 \times 5$ & 90 & 5 \\
\hline $5^{\text {th }}$ week & $4 \times 5$ & 90 & 5 \\
\hline \multicolumn{4}{|c|}{ Evaluation of eccentric power output } \\
\hline $6^{\text {th }}$ week & $5 \times 6$ & 90 & 6 \\
\hline $7^{\text {th }}$ week & $5 \times 6$ & 90 & 6 \\
\hline \multicolumn{4}{|c|}{ Evaluation of eccentric power output } \\
\hline $8^{\text {th }}$ week & $6 \times 6$ & 90 & 7 \\
\hline
\end{tabular}

\subsection{Electromyography}

EMG activity was recorded on the dominant leg with surface bipolar $(3.5 \mathrm{~cm}$ center to center) electrodes (Quinton Quick prep silver/silver chloride) over the rectus femoris, the long head of biceps femoris and the medialis gastrocnemius muscles. These muscles were selected as main contributors to perform vertical jumps [21, 22]. Electrodes were placed according to the SENIAM guidelines [23]. Skin was properly shaved and cleaned with alcohol solution to keep skin impendence below $5 \mathrm{k} \Omega$. To ensure consistent placement of the electrodes for each testing session, marks was placed around the circumference of the electrodes with a permanent marker and accordingly the placed electrodes were photographed. For the rectus femoris the electrodes were placed at midpoint between the anterior spina iliaca superior and the superior part of the patella. The biceps femoris electrodes were attached at the half distance between the ischial tuberosity and the lateral epicondyle of the tibia. For the gastrocnemius the electrodes were placed on the gastrocnemius medialis on the most prominent bulge of the muscle. During the assessments the electrodes were not removed and relocated on the subjects' muscles.

The signal was pre-amplified (gain $=\mathrm{X} 400$ ) and sampled at $1000 \mathrm{~Hz}$. Raw EMG was band pass filtered from 8 to $500 \mathrm{~Hz}$ with a Butterworth 4th order filter and the root mean square (RMS) for a $20 \mathrm{~ms}$ time window was calculated with custom Matlab scripts (version 7, The MathWorks Inc.). According to De Louca [24], the RMS (square root of the integral of the square of the EMG potential with respect to time), has a physical meaning and provides a better strength signal in dynamic muscle activity volitionally produced. The mean of the processed signal was calculated i) during the braking and propulsion phase of the counter movement jump, ii) $100 \mathrm{~ms}$ before contact (pre-activation) [25], and during the braking and propulsion of the drop jump, iii) during the whole range of motion throughout the eccentric and concentric phase of the isokinetic movement. All raw signals were synchronized, digitized and saved on an A/D card (AMTI 32channel analog data version 2.02).

\subsection{Kinematic Data}

The kinematic data during the jumps were analyzed using the 2D Ariel Performance Analysis System (APAS) while the motion captured with a digital camera (Panasonic, PV-900). Five reflective markers (14 $\mathrm{mm}$ spheres) were placed at anatomical bony landmarks (i.e. acromion, femoral epicondyle, lateral tibia, lateral malleolus and $5^{\text {th }}$ metatarsal head).

\subsection{Statistical Analysis}

Two-way ANOVA [group (training and control group) $\times$ week (first and eighth week)] with repeated measurements on time were used. If a significant interaction was obtained, pairwise comparisons were performed through simple main effect analysis. t-test was used to compare the anthropometric characteristics between the eccentric training and the control groups. Data are presented as mean \pm SEM while the level of significance was set at $\alpha=0.05$. The SPSS version 15.0 was used for all analyses (SPSS Inc., Chicago, Illinois).
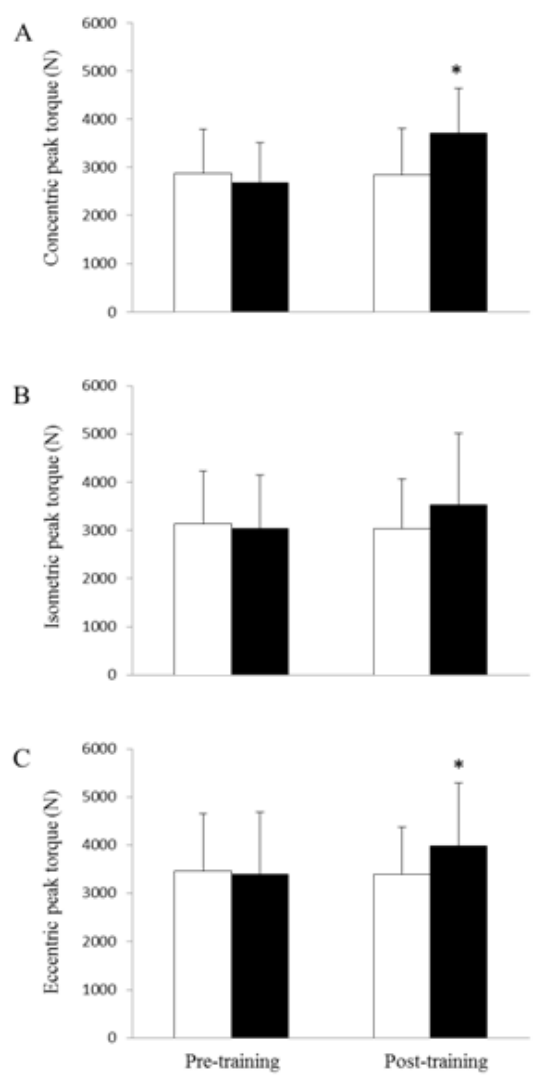

Figure 2. Concentric (A), isometric (B) and eccentric (C) peak torque pre and post eccentric training for the control $(\square)$ and the experimental group ( $\boldsymbol{\square})$. *significant different $(p<0.05)$ between pre and post training 

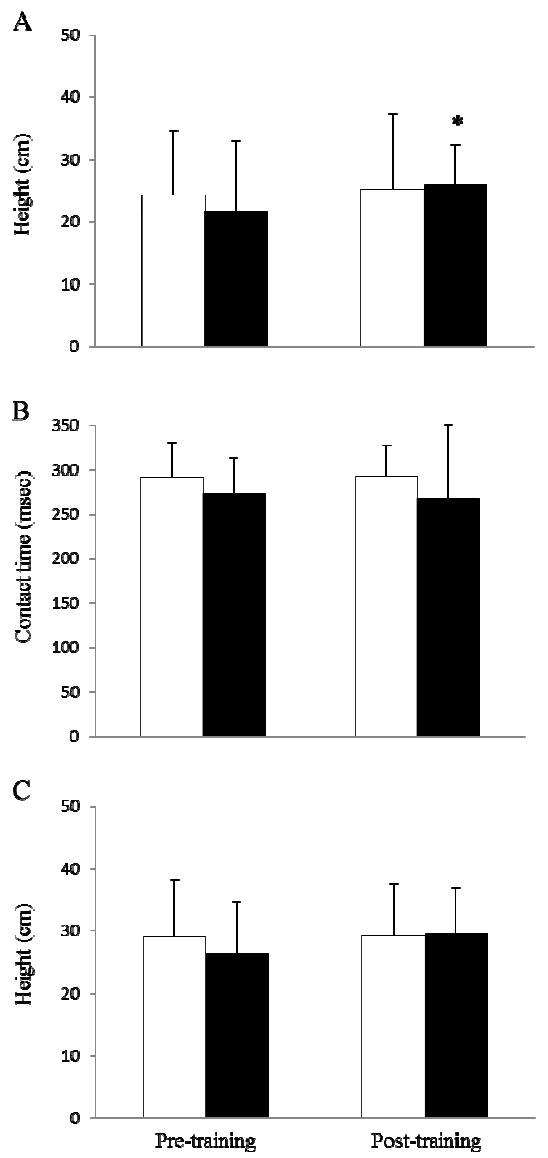

Figure 3. Height (A) and contact time (B) during drop jump and height during counter movement jump $(C)$ pre and post eccentric training for the control ( $\square$ ) and the experimental group ( $\mathbf{\square})$. *significant different ( $p<0.05)$ between pre and post training

\section{Results}

No significant differences in anthropometric characteristics were observed between the two groups. After 8 weeks of eccentric training using a multi-joint isokinetic dynamometer concentric and eccentric muscle strength was elevated only in the experimental group (Figure 2). Regarding the jumping ability no significant alterations were observed in counter movement and the in the contact time of the drop jump for both the control and the experimental group (Figure 3). However, in the experimental group, eccentric training cause elevation in height performance during the drop jumps (Figure 3). Additionally, after the eccentric training, the hip flexion and the dorsiflexion were significantly reduced. Additionally, during the takeoff phase of the drop jump, eccentric training reduced significantly the hip and the ankle angles to $149^{\circ}$ from $154^{\circ}$ and to $100^{\circ}$ from $105^{\circ}$, respectively. The control group at the takeoff phase of the first and the second assessment revealed for the hip joint $144^{\circ}$ and $143^{\circ}$ respectively and for the angle joint $103^{\circ}$ and $106^{\circ}$ respectively.

After the eight weeks of eccentric training, EMG activity during counter movement jump significantly elevated in rectus femoris and significantly reduced in biceps femoris during the propulsive phase while EMG activity did not alter during the braking phase (Table 2). After the training period, EMG activity during drop jump significantly elevated during pre-activation phase only in gastrocnemius muscle (Table 2). After the eccentric training the EMG activity during drop jump did not alter in any of the investigated muscles during the braking and propulsive phases.

Table 2. EMG activity $(\mathrm{mV})$ during the eccentric and the concentric phase of CMJ as well as during the pre-activation, the eccentric and the concentric phase of $D J$.

\begin{tabular}{|c|c|c|c|c|}
\hline & \multicolumn{2}{|c|}{ Control Group } & \multicolumn{2}{|c|}{ Experimental Group } \\
\hline & Pre-training & Post-training & Pre-training & Post-training \\
\hline \multicolumn{5}{|l|}{$\mathrm{CMJ}$} \\
\hline \multicolumn{5}{|c|}{ Breaking phase } \\
\hline $\mathrm{RF}$ & $0.118 \pm 0.053$ & $0.080 \pm 0.026$ & $0.100 \pm 0.056$ & $0.095 \pm 0.035$ \\
\hline $\mathrm{BF}$ & $0.071 \pm 0.089$ & $0.173 \pm 0.197$ & $0.110 \pm 0.104$ & $0.032 \pm 0.006$ \\
\hline GAS & $0.040 \pm 0.006$ & $0.051 \pm 0.019$ & $0.053 \pm 0.035$ & $0.071 \pm 0.043$ \\
\hline \multicolumn{5}{|c|}{ Propulsive phase } \\
\hline $\mathrm{RF}$ & $0.300 \pm 0.065$ & $0.185 \pm 0.086$ & $0.204 \pm 0.137$ & $0.263 \pm 0.168^{*}$ \\
\hline $\mathrm{BF}$ & $0.158 \pm 0.088$ & $0.372 \pm 0.352$ & $0.184 \pm 0.038$ & $0.072 \pm 0.039$ \\
\hline GAS & $0.219 \pm 0.058$ & $0.223 \pm 0.071$ & $0.204 \pm 0.079$ & $0.237 \pm 0.120$ \\
\hline \multicolumn{5}{|l|}{ DJ } \\
\hline \multicolumn{5}{|c|}{ Pre-activation } \\
\hline $\mathrm{RF}$ & $0.108 \pm 0.060$ & $0.064 \pm 0.010$ & $0.081 \pm 0.051$ & $0.109 \pm 0.097$ \\
\hline $\mathrm{BF}$ & $0.074 \pm 0.043$ & $0.071 \pm 0.044$ & $0.055 \pm 0.020$ & $0.039 \pm 0.015$ \\
\hline GAS & $0.149 \pm 0.081$ & $0.158 \pm 0.087$ & $0.123 \pm 0.064$ & $0.207 \pm 0.067$ \\
\hline \multicolumn{5}{|c|}{ Breaking phase } \\
\hline $\mathrm{RF}$ & $0.388 \pm 0.180$ & $0.190 \pm 0.115$ & $0.205 \pm 0.145$ & $0.201 \pm 0.185$ \\
\hline $\mathrm{BF}$ & $0.220 \pm 0.378$ & $0.189 \pm 0.134$ & $0.183 \pm 0.084$ & $0.079 \pm 0.031$ \\
\hline GAS & $0.171 \pm 0.087$ & $0.195 \pm 0.087$ & $0.584 \pm 1.250$ & $0.210 \pm 0.109$ \\
\hline \multicolumn{5}{|c|}{ Propulsive phase } \\
\hline $\mathrm{RF}$ & $0.344 \pm 0.170$ & $0.172 \pm 0.085$ & $0.222 \pm 0.134$ & $0.149 \pm 0.099$ \\
\hline $\mathrm{BF}$ & $0.171 \pm 0.077$ & $0.403 \pm 0.527$ & $0.188 \pm 0.083$ & $0.108 \pm 0.063$ \\
\hline GAS & $0.242 \pm 0.083$ & $0.214 \pm 0.083$ & $0.468 \pm 0.856$ & $0.333 \pm 0.137$ \\
\hline
\end{tabular}

$\mathrm{BF}$, biceps femoris; CMJ, counter movement jump; DJ, drop jump; GAS, gastrocnemius; RF, rectus femoris* significant different ( $<<0.05$ ) between pre and post training. 
After the eight weeks of eccentric training, the EMG activity during the eccentric force assessment, was found to be significantly increased in rectus femoris and significant decreased in gastrocnemius while did not affect in biceps femoris (Table 3). No differences in EMG activity were observed between the control and the experimental group while in the control group no differences were observed between the pre and post training assessments (Table 2).

Table 3. EMG activity $(\mathrm{mV})$ during maximal eccentric power output of rectus femoris (pre-activation phase), biceps femoris and gastrocnemius (activation phase).

\begin{tabular}{lll}
\hline & Control group & Experimental group \\
\hline $\begin{array}{l}\text { Rectus femoris } \\
\text { Pre-training }\end{array}$ & $0.057 \pm 0.054$ & $0.027 \pm 0.070$ \\
Post-training & $0.056 \pm 0.052^{\#}$ & $0.096 \pm 0.018^{*}$ \\
Biceps femoris & & \\
Pre-training & $0.061 \pm 0.049$ & $0.024 \pm 0.069$ \\
Post-training & $0.060 \pm 0.042$ & $0.069 \pm 0.033$ \\
Gastrocnemius & & \\
Pre-training & $0.074 \pm 0.069^{\#}$ & $0.172 \pm 0.044$ \\
Post-training & $0.075 \pm 0.068$ & $0.056 \pm 0.128^{*}$ \\
\hline
\end{tabular}

*significant different $(\mathrm{p}<0.05)$ between pre and post training

\#significant different $(\mathrm{p}<0.05)$ between the control and experimental group

\section{Discussion}

The purpose of the present investigation was to evaluate the effects of eccentric training using a multi-joint dynamometer on EMG activity of rectus femoris, biceps femoris, as well as medial gastrocnemius during counter movement jump, drop jump and during maximal eccentric action. As it was expected the control group did not reveal any differences between the pre and post training strength assessment, jumping ability, and EMG activity. On the contrary, in the experimental group the eccentric training affected several of the examined parameters. Specifically, there was significant elevation of concentric and eccentric force while the height of the drop jump was also elevated after the training period. The EMG activity of the examined muscles altered during counter movement jump, drop jump and eccentric isokinetic assessment. However, no difference was observed between the control and the experimental group before or after the eccentric training period.

Isokinetic dynamometers are used in most investigations where eccentric exercise has to be performed. The muscle group that contracts during exercise is isolated in all these isokinetic dynamometers. However, the majority of human movements are multi-joint and recruit a larger number of muscles where agonists enhance movement whereas the co-activation of antagonists results in restriction of speed and amplitude of movement [26]. Taking into account in the present investigation the limitations of the commercially available isokinetic dynamometers, the training protocol as well as the strength assessments were performed on a custom-built multi-joint isokinetic type machine. This device was designed to measure the amount of external work and the linear velocity on the whole range of motion during concentric and eccentric exercise modes.

It is known that eccentric training is associated with higher forces production [27] and larger strength improvements [28] compared to isometric or concentric training. Indeed, in a study where 8 weeks of eccentric or concentric training was performed, eccentric training induced greater increases in baseline eccentric and isometric torque compared to concentric training [29]. Authors suggested that the specific physiological adaptations to resistance exercise are determined by the muscle actions involved $[29,30]$. The present finding are in line with previous investigations given the fact that 8 weeks of eccentric training induced notable elevations of peak force compared to the control group.

This could be attributed to the rather lower intensity and the limited number of repetitions that were adopted in the present investigation especially in the first weeks of the training period. Additionally, the fact that the subjects of the control group continued to participate in their regular training may also account for the absence of differences compared to the experimental group.

Eccentric exercise is known to induce modulations in the recruitment order, discharge rate and recruitment threshold of the motor units [31]. It is also known that during an eccentric contraction, the electric activity of a muscle is lower than the one recorded during a concentric contraction for the same level of the force produced [32-34]. However, eccentric training can increase this neuromuscular activation $[33,35]$. These could be the case in the present investigation, where the eccentric training, caused increases in EMG activity in the pre-activation phase of gastrocnemius during drop jump which could be further attributed to favorable changes in reflex excitability leading to better facilitation of the myotatic reflex and to depression of the autogenic inhibition generated by the Golgi tendon organs [35]. Additionally, during the takeoff phase of the drop jump, it was found that hip and the ankle angles were reduced. It is suggested that the smaller joint angles are induced by the eccentric training which may cause changes in the architecture in muscle tendon unit [36] and affect both proprioception, force-length and force-velocity relationship [5, 18].

During the counter movement jump, the elevated EMG activity of rectus femoris and the reduced EMG activity of the biceps femoris indicate that eccentric training improved the relationship between agonists and antagonists in favor for the first. Moreover, during the post training eccentric assessment the EMG activity of the rectus femoris and medial gastrocnemius found to be elevated. Indeed, it is known that repeated eccentric training could lead to a progressive recruitment of slow motor units for the better 
distribution of mechanical stress and may also lead to increase in the discharge frequency of motor units $[16,18$, 37, 38]. Caution should be paid however, since the lower EMG activation of the antagonist muscles may induce injuries, since their function is to protect the joint and muscle structures against excessively high tension levels that may occur during eccentric actions [10].

\subsection{Conclusion}

It is a fact that coaches and athletes seek ways to increase the effectiveness of conditioning programs while eccentric training seems to be the best way for maximizing strength performance. Indeed, eccentric exercise induces structural changes on the muscle-tendon system and adaptations of the nervous central command, causing increases in maximal force production, often higher to those obtained with concentric or isometric training [16]. In the present investigation, it is clear that eccentric training using a multi-joint isokinetic dynamometer may exerts favorable effects of muscle strength and EMG activity of the agonist muscles in amateur young soccer players. Moreover, in competitive sports most muscle strain injuries occur during eccentric muscle actions, and then the training of muscle groups using eccentric exercise may help to diminish the risk of injuries [39]. More research is needed in the area of eccentric training in young athletes using multi-joint isokinetic dynamometers.

\section{References}

[1] D. W. Chapman, M. J. Newton, Z. Zainuddin, P. Sacco, and K. Nosaka. Work and peak torque during eccentric exercise do not predict changes in markers of muscle damage. $\mathrm{Br} \mathrm{J}$ Sports Med. 2008, 42, 585-91.

[2] A. P. Lavender, and K. Nosaka. Changes in fluctuation of isometric force following eccentric and concentric exercise of the elbow flexors. Eur J Appl Physiol. 2006, 96, 235-40.

[3] L. Norrbrand, J. D. Fluckey, M. Pozzo, and P. A. Tesch. Resistance training using eccentric overload induces early adaptations in skeletal muscle size. Eur J Appl Physiol. 2008, $102,271-81$

[4] V. Paschalis, Y. Koutedakis, A. Z. Jamurtas, V. Mougios, and V. Baltzopoulos. Equal volumes of high and low intensity of eccentric exercise in relation to muscle damage and performance. J Strength Cond Res. 2005, 19, 184-8.

[5] T. C. Chen, K. Nosaka, and P. Sacco. Intensity of eccentric exercise, shift of optimum angle, and the magnitude of repeated-bout effect. J Appl Physiol. 2007, 102, 992-9.

[6] V. Paschalis, M. G. Nikolaidis, G. Giakas, A. Z. Jamurtas, E. O. Owolabi, and Y. Koutedakis. Position sense and reaction angle after eccentric exercise: the repeated bout effect. Eur J Appl Physiol. 2008, 103, 9-18.

[7] J. Duclay, A. Martin, A. Robbe, and M. Pousson. Spinal reflex plasticity during maximal dynamic contractions after eccentric training. Med Sci Sports Exerc. 2008, 40, 722-34.
[8] A. Nardone, C. Romano, and M. Schieppati. Selective recruitment of high-threshold human motor units during voluntary isotonic lengthening of active muscles. J Physiol. 1989, 409, 451-71.

[9] M. Roig, K. O'Brien, G. Kirk, R. Murray, P. McKinnon, B. Shadgan, and W. D. Reid. The effects of eccentric versus concentric resistance training on muscle strength and mass in healthy adults: a systematic review with meta-analysis. Br J Sports Med. 2009, 43, 556-68.

[10] E. Kellis, and V. Baltzopoulos. Isokinetic eccentric exercise. Sports Med. 1995, 19, 202-22.

[11] M. C. Kostek, Y. W. Chen, D. J. Cuthbertson, R. Shi, M. J. Fedele, K. A. Esser, and M. J. Rennie. Gene expression responses over $24 \mathrm{~h}$ to lengthening and shortening contractions in human muscle: major changes in CSRP3, MUSTN1, SIX1, and FBXO32. Physiol Genomics. 2007, 31, 42-52.

[12] S. Hody, P. Leprince, K. Sergeant, J. Renaut, J. L. Croisier, F. Wang, and B. Rogister. Human muscle proteome modifications after acute or repeated eccentric exercises. Med Sci Sports Exerc. 2011, 43, 2281-96.

[13] H. Langberg, H. Ellingsgaard, T. Madsen, J. Jansson, S. P. Magnusson, P. Aagaard, and M. Kjaer. Eccentric rehabilitation exercise increases peritendinous type I collagen synthesis in humans with Achilles tendinosis. Scand J Med Sci Sports. 2007, 17, 61-6.

[14] K. Falconer, and D. A. Winter. Quantitative assessment of co-contraction at the ankle joint in walking. Electromyogr Clin Neurophysiol. 1985, 25, 135-49.

[15] M. Solomonow, R. Baratta, B. H. Zhou, and R. D'Ambrosia. Electromyogram coactivation patterns of the elbow antagonist muscles during slow isokinetic movement. Exp Neurol. 1988, 100, 470-7.

[16] G. Guilhem, C. Cornu, and A. Guevel. Neuromuscular and muscle-tendon system adaptations to isotonic and isokinetic eccentric exercise. Ann Phys Rehabil Med. 2010, 53, 319-41.

[17] M. Pensini, A. Martin, and N. A. Maffiuletti. Central versus peripheral adaptations following eccentric resistance training. Int J Sports Med. 2002, 23, 567-74.

[18] S. Colson, M. Pousson, A. Martin, and J. Van Hoecke. Isokinetic elbow flexion and coactivation following eccentric training. J Electromyogr Kinesiol. 1999, 9, 13-20.

[19] C. A. Moore, L. W. Weiss, B. K. Schilling, A. C. Fry, and Y. Li. Acute effects of augmented eccentric loading on jump squat performance. J Strength Cond Res. 2007, 21, 372-7.

[20] D. Paddon-Jones, M. Leveritt, A. Lonergan, and P. Abernethy. Adaptation to chronic eccentric exercise in humans: the influence of contraction velocity. Eur J Appl Physiol. 2001, $85,466-71$.

[21] M. F. Bobbert, and G. J. van Ingen Schenau. Coordination in vertical jumping. J Biomech. 1988, 21, 249-62.

[22] A. J. Van Soest, A. L. Schvab, M. F. Bobbert, and G. L. van Ingen Schenau. The influence of bi-articularity of the gastrocnemius muscle on vertical jumping achievement. J Biomech. 1993, 26, 1-8. 
[23] H. J. Hermens, B. Frerics, C. Disselhorst-Klug, and G. Rau. Development of recommendations for SEMG sensors and sensor placement procedures. J Electromyogr Kinesiol. 2000, $10,361-74$

[24] C. J. De Luca. The use of surface electromyography in Biomechanics. Journal of Applied Biomechanics. 1997, 13, 135-63.

[25] T. Horita, P. V. Komi, C. Nicol, and H. Kyrolainen. Interaction between pre-landing activities and stiffness regulation of the knee joint musculoskeletal system in the drop jump: implications to performance. Eur J Appl Physiol. $2002,88,76-84$.

[26] C. D. Marsden, J. A. Obeso, and J. C. Rothwell. The function of the antagonist muscle during fast limb movements in man. J Physiol. 1983, 335, 1-13.

[27] S. H. Westing, A. G. Cresswell, and A. Thorstensson. Muscle activation during maximal voluntary eccentric and concentric knee extension. Eur J Appl Physiol Occup Physiol. 1991, 62, 104-8.

[28] T. Hortobagyi, J. Barrier, D. Beard, J. Braspennincx, P. Koens, P. Devita, L. Dempsey, and J. Lambert. Greater initial adaptations to submaximal muscle lengthening than maximal shortening. J Appl Physiol. 1996, 81, 1677-82.

[29] V. Paschalis, M. G. Nikolaidis, A. A. Theodorou, G. Panayiotou, I. G. Fatouros, Y. Koutedakis, and A. Z. Jamurtas A weekly bout of eccentric exercise is sufficient to induce health-promoting effects. Med Sci Sports Exerc. 2011, 43, 64-73.

[30] G. A. Dudley, P. A. Tesch, B. J. Miller, and P. Buchanan. Importance of eccentric actions in performance adaptations to resistance training. Aviat Space Environ Med. 1991, 62, $543-50$

[31] R. M. Enoka. Eccentric contractions require unique activation strategies by the nervous system. J Appl Physiol.
$1996,81,2339-46$.

[32] P. Aagaard, E. B. Simonsen, J. L. Andersen, S. P. Magnusson, J. Halkjaer-Kristensen, and P. Dyhre-Poulsen. Neural inhibition during maximal eccentric and concentric quadriceps contraction: effects of resistance training. J Appl Physiol. 2000, 89, 2249-57.

[33] P. V. Komi, and E. R. Buskirk. Effect of eccentric and concentric muscle conditioning on tension and electrical activity of human muscle. Ergonomics. 1972, 15, 417-34.

[34] V. Linnamo, T. Moritani, C. Nicol, and P. V. Komi. Motor unit activation patterns during isometric, concentric and eccentric actions at different force levels. J Electromyogr Kinesiol. 2003, 13, 93-101.

[35] T. Hortobagyi, J. P. Hill, J. A. Houmard, D. D. Fraser, N. J. Lambert, and R. G. Israel. Adaptive responses to muscle lengthening and shortening in humans. J Appl Physiol. 1996, $80,765-72$.

[36] N. N. Mahieu, P. McNair, A. Cools, C. D'Haen, K. Vandermeulen, and E. Witvrouw. Effect of eccentric training on the plantar flexor muscle-tendon tissue properties. Med Sci Sports Exerc. 2008, 40, 117-23.

[37] C. Bosco, J. T. Viitasalo, P. V. Komi, and P. Luhtanen. Combined effect of elastic energy and myoelectrical potentiation during stretch-shortening cycle exercise. Acta Physiol Scand. 1982, 114, 557-65.

[38] V. Linnamo, R. Bottas, and P. V. Komi. Force and EMG power spectrum during and after eccentric and concentric fatigue. J Electromyogr Kinesiol. 2000, 10, 293-300.

[39] T. W. Kaminski, C. V. Wabbersen, and R. M. Murphy. Concentric versus enhanced eccentric hamstring strength training: clinical implications. J Athl Train. 1998, 33, 216-21. 